\title{
Article
}

\section{Selective Sc Recovery from Rare Earths in Nitric Acid Medium by Extraction Chromatography}

\author{
Sou WATANABE ${ }^{1 *}$, Hideya SUZUKI ${ }^{2}$, Ichiro GOTO ${ }^{1}$, Hirohide KOFUJI ${ }^{1}$ \\ and Tatsuro MATSUMURA ${ }^{2}$ \\ ${ }^{1}$ Nuclear Fuel Cycle Engineering Laboratories, Japan Atomic Energy Agency, 4-33, Muramatsu, Tokai-mura, \\ Naka-gun, Ibaraki, Japan \\ ${ }^{2}$ Nuclear Science Research Institute, Japan Atomic Energy Agency, 2-4, Shirakata, Tokai-mura, \\ Naka-gun, Ibaraki, Japan
}

(Manuscript received April 9, 2018; accepted June 19, 2018)

\begin{abstract}
Chemical compounds containing scandium $(\mathrm{Sc})$ are widely applied to various fields such as catalysts, alloys, lamps and etc. Sc is found in mineral ore, and it is necessary to develop efficient Sc separation and recovery technology for the industrial applications. $N, N, N^{\prime}, N^{\prime}, N$,', $N$ ''-hexaoctylnitrilotriacetamide (HONTA) extractant has been shown to be promising for selective Sc extraction. In this study, applicability of the extraction chromatography technology using HONTA impregnated adsorbent for the selective Sc recovery from other rare earth elements (REs) was experimentally evaluated through batch-wise adsorption/elution studies and column separation experiments. Batch-wise experiments showed that distribution coefficient of Sc onto the $\mathrm{HONTA} / \mathrm{SiO}_{2}-\mathrm{P}$ adsorbent was far larger than those of other REs at acidity region with $0.001<\left[\mathrm{HNO}_{3}\right]<4 \mathrm{~mol} \mathrm{dm}^{-3}$ and that the adsorbed Sc was efficiently eluted into $1 \mathrm{M}$ $\mathrm{H}_{2} \mathrm{SO}_{4}$ solution. Appropriate experimental conditions for the column separation experiment were proposed based on those results, and performance of the flow-sheet was examined for laboratory scale column system. Only Sc in $4 \mathrm{~mol} \mathrm{dm}{ }^{-3} \mathrm{HNO}_{3}$ solution was adsorbed into a packed column with the adsorbent while other REs were discharged from the column with the feed solution, and then loaded Sc was properly eluted into $1 \mathrm{M} \mathrm{H}_{2} \mathrm{SO}_{4}$ solution. Purity of the Sc product solution obtained by the column separation experiment depended on Sc recovery ratio, and about $99.9 \%$ purity was achieved with $97 \%$ recovery ratio. The residual Sc was eluted from the column with other REs and did not remain inside the column, therefore repeated used of the column was shown to be possible. This process was shown to be promising for selective Sc recovery from mixture of REs in nitric acid.
\end{abstract}

Keywords: Scandium recovery, Rare earth elements, Extraction chromatography, HONTA

\section{Introduction}

Wide variety of compounds containing scandium $(\mathrm{Sc})$ are recently attracted for various applications such as catalyst ${ }^{1,2)}$, alloys $^{3,4)}$, lamp of laser ${ }^{5)}$ and etc. Rare earth elements (REs) involving 15 lanthanides, yttrium and Sc are chemically similar, therefore the yield of scandium is not so large due to difficulty in separation from other REs. Effective and appropriate Sc extraction and purification procedures from mineral ore, which is main source of $\mathrm{Sc}$, is essential for its commercial use. So far, several fundamental studies on recovery and purification

\footnotetext{
* Corresponding author

E-mail: watanabe.sou@jaea.go.jp
} 
technologies of scandium have been carried out ${ }^{6-9)}$. However, an efficient process with releasing small amount of secondary wastes is desirable for practical operation. Our group is focusing on the extraction chromatography as a promising technology for separation and purification of Sc from chemically similar elements due to its simplicity in operation and compactness in equipment.

Japan Atomic Energy Agency has been developing the extraction chromatography technology for recovery of trivalent minor actinides (MA(III): Am and $\mathrm{Cm}$ ) from spent nuclear fuel, and performance of the technology has been systematically investigated so $\mathrm{far}^{10-15)}$. The technology employs porous silica based adsorbent impregnating an extractant in the particle, and adsorption/elution reactions inside the packed column enable to separate and recover target elements involved in a feed solution. The porous silica particle with about $50 \mu \mathrm{m}$ diameter and $50 \mathrm{~nm}$ pore diameter are coated by styrene divinyl benzene copolymer (referred as $\mathrm{SiO}_{2}-\mathrm{P}$ ), and various extractants can be utilized for the adsorbent. The extractant is adopted according to the target element, and an appropriate flow-sheet is designed based on fundamental adsorption/elution performance of the adsorbent. This technology can reduce amount of secondary wastes compared to the solvent extraction process since it does not require any diluent and the number of stages can be easily increased not by adding equipment but by simply lengthening the column. Small difference in distribution coefficient between elements onto the adsorbent is fully utilized for the separation of them by modifying operation conditions. These features are suitable for practical Sc recovery from REs mixtures.

Recent development in the extractant for MA(III) recovery has found that $N, N, N^{\prime}, N$,,$N$ ', $N$ ''-hexaoctylnitrilotriacetamide $\left(\right.$ HONTA, Fig. 1) ${ }^{16)}$ extractant which show selectivity for MA(III) over REs has strong affinity to Sc. In this study, HONTA impregnated adsorbent (HONTA/ $\mathrm{SiO}_{2}-\mathrm{P}$ ) was prepared, and applicability of the adsorbent to the extraction chromatography for Sc recovery was experimentally evaluated. Batch-wise adsorption/elution experiments were carried out to evaluate influence of acidity on the distribution coefficients of REs and to give an appropriate flow-sheet for column separation operation. Based on the fundamental adsorption/elution data, the flow-sheet was proposed and its performance was evaluated through recovery ratio and purity of $\mathrm{Sc}$ in a product solution obtained by a laboratory scale column separation experiment.

\subsection{Preparation of adsorbent}

\section{Experimental}

Porous silica particle with about $50 \mathrm{~nm}$ pore size and about $50 \mu \mathrm{m}$ particle diameters were prepared by sol-gel method. Porosity of the particle was about 0.35 . Styrene-divinyl benzene copolymer was applied on surface of the particle with about several hundred nanometers thickness ${ }^{17)}$. Degree of the cross

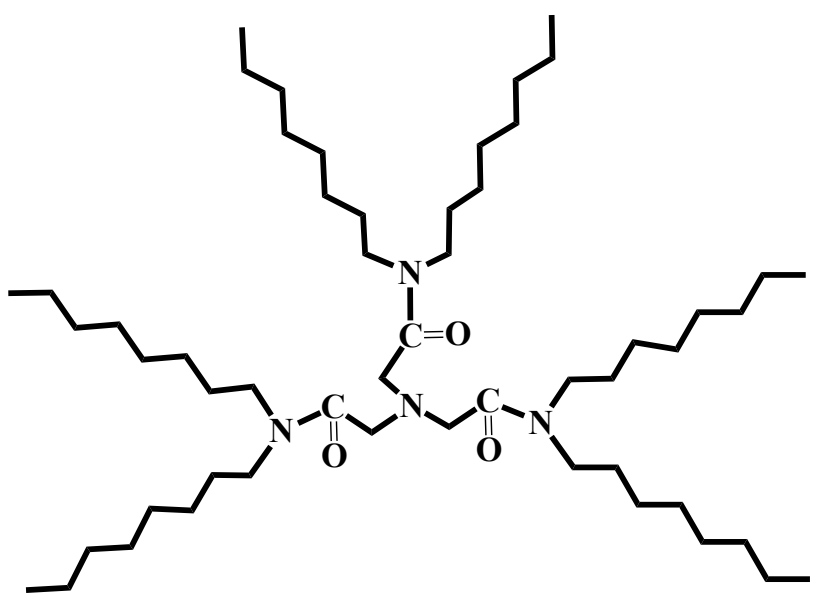

Fig. $1 N, N, N^{\prime}, N^{\prime}, N$ ', $N$ ''-hexaoctyInitrilotriacetamide (HONTA)

linkage of the copolymer was $15 \%$. HONTA extractant were impregnated into the polymer. Amount of extractants impregnated was fixed at $33 \mathrm{wt} \%$ of total weight of the adsorbent. Detail preparation procedure is described in a reference ${ }^{18)}$.

\subsection{Batch-wise experiments}

$0.5 \mathrm{~g}$ of the adsorbent was mixed with $10 \mathrm{~mL}$ of the feed solution containing $20 \mathrm{mg} / \mathrm{L}$ of all rare earth elements (except for Pm(III)), and then shaken for $3 \mathrm{~h}$ at $298 \mathrm{~K}$. Acidity of the feed solution was parametrically changed at $0.001<\left[\mathrm{HNO}_{3}\right]<4$ mol $\mathrm{dm}^{-3}(\mathrm{M})$. After separating solid and liquid phases, concentrations of the rare earth elements in the supernatant solution were analyzed by ICP-AES measurement.

Batch-wise elution experiments were also carried out using REs loaded adsorbent for DTPA, citric acid and sulfuric acid solutions as eluents. $0.2 \mathrm{~g}$ of the adsorbent after contacting with the feed solution was mixed with $4 \mathrm{~mL}$ of the eluents, and then shaken for $3 \mathrm{~h}$ at $298 \mathrm{~K}$. Acidity of the feed solution was decided based on the results of the adsorption experiments. Elution ratios of REs were calculated by these experiments, and then an appropriate elution condition for the column separation experiment was determined.

\subsection{Column separation experiments}

Column separation experiments were carried out with a condition derived from the batch-wise experiments. Experimental setup consisted of a column, a pump for feeding solutions, a switching valve and a fraction collector. HONTA $/ \mathrm{SiO}_{2}-\mathrm{P}$ adsorbent was packed in a glass column with 1 $\mathrm{cm} \phi \times 10 \mathrm{~cm}$ height $\left(1 \mathrm{Bed}\right.$ Volume $\left.(\mathrm{BV})=7.85 \mathrm{~cm}^{3}\right)$. Feed solution, wash solution and an eluent were sequentially supplied into the column, and then effluent was fractionally collected at every 0.5 BV. Concentrations of REs in the fractions were analyzed by ICP-AES measurement. Elution curves of REs were obtained, and recovery ratio of Sc and purity of Sc in a product solution were calculated. 


\section{Results and discussion}

\subsection{Batch-wise experiments}

Distribution coefficients $K_{\mathrm{d}}$ of REs onto $\mathrm{HONTA} / \mathrm{SiO}_{2}-\mathrm{P}$ adsorbent calculated by the following equation are shown in Fig. 2;

$$
K_{d}=\frac{C_{0}-C_{s}}{C_{0}} \times \frac{V}{W}
$$

where $C_{0}$ and $C_{\mathrm{s}}$ are concentrations of cations in the solution before and after the adsorption, respectively, $V$ is volume of the solution, $W$ is weight of the adsorbent.

The HONTA/ $/ \mathrm{SiO}_{2}-\mathrm{P}$ selectively adsorbed $\mathrm{Sc}$ and $K_{\mathrm{d}}$ of $\mathrm{Sc}$ was far larger than those of other REs at any acidity region although $K_{\mathrm{d}}$ has local minimum at $\left[\mathrm{HNO}_{3}\right]=2 \mathrm{M}$. Selective adsorption of Sc from nitric acid solution containing all REs should be possible. The distinct difference in the $K_{\mathrm{d}}$ between different RE elements might be caused by difference in ionic radii of them. Hydration behavior of $\mathrm{Sc}(\mathrm{III})$ is reported to be different from those of other REs due to its small size ${ }^{19)}$.

$K_{\mathrm{d}}$ for all REs drastically depended on acidity of the feed solution, and those variation might be attributed to contribution of $\mathrm{NO}_{3}{ }^{-}$ions for formation of complexes. These features have been observed also in the solvent extraction system ${ }^{16)}$, and selective $\mathrm{MA}(\mathrm{III})$ adsorption onto the $\mathrm{HONTA} / \mathrm{SiO}_{2}-\mathrm{P}$ is expected at $\left[\mathrm{HNO}_{3}\right]=0.1 \mathrm{M}$ according to the distribution behavior into the solvent. Design of MA(III) recovery flowsheet from spent nuclear fuel is in progress, and performance of the flow-sheet will be examined in near future.

In order to achieve selective Sc adsorption with suppressing extraction of other REs by the HONTA, an appropriate acidity of the feed solution for the column separation operation was decided to be $4 \mathrm{M}$. In this condition, almost REs except for La and $\mathrm{Ce}$ were not loaded onto the adsorbent. The batch-wise elution experiments were carried out using the condition, and elution ratio of only $\mathrm{Sc}$ was evaluated. Figure 3 shows the elution ratios of $\mathrm{Sc}$ from the adsorbent onto various solutions. Complex reagents such as citric acid and DTPA in nitric acid solution effectively worked for Sc stripping, however sulfuric acid achieved the highest elution ratio. Elution ratio increased with increasing the acidity at low acidity region, whereas elution ratio decreased at $\left[\mathrm{H}_{2} \mathrm{SO}_{4}\right]>1 \mathrm{M}$. The ionic strength of the eluent is considered to influence on the elution behavior. An appropriate acidity of the eluent for the column separation was set to be $1 \mathrm{M}$.

\subsection{Column separation experiments}

Experimental conditions of the column separation are shown in Table 1, where concentration of each REs in the feed solution was $100 \mathrm{ppm}$. After conditioning the packed column with $4 \mathrm{M}$ $\mathrm{HNO}_{3}$ solution, $25 \mathrm{BV}$ of the feed solution was fed into the column. Amount of the supplied Sc was one third of the adsorption capacity of $\mathrm{HONTA} / \mathrm{SiO}_{2}-\mathrm{P}$ packed column assuming that one HONTA molecule forms complex with one

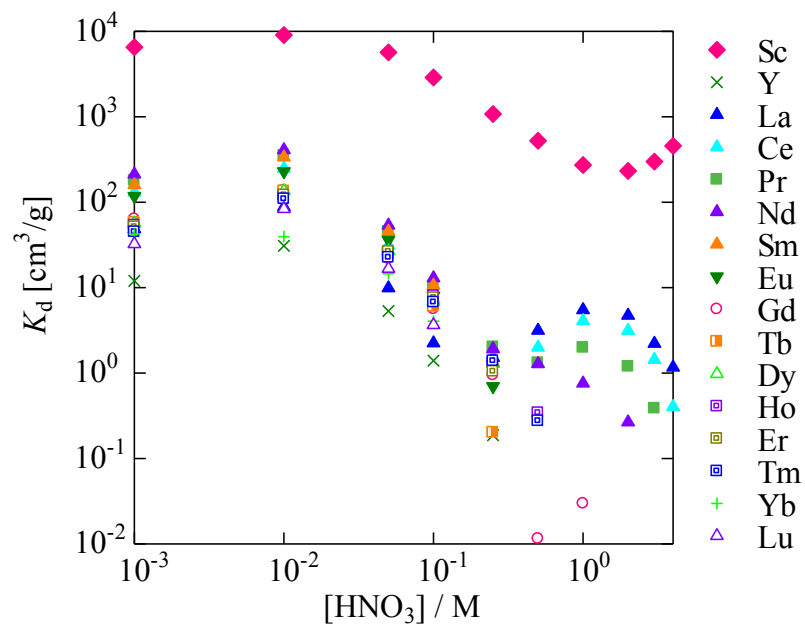

Fig. 2 Distribution coefficient of RE(III) onto HONTA/SiO ${ }_{2}-\mathrm{P}$ adsorbent.

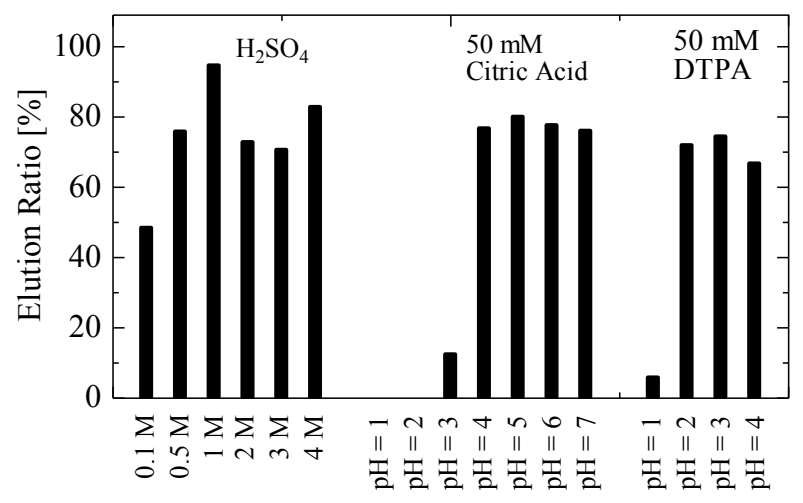

Fig. 3 Elution ratio of Sc from $\mathrm{HONTA} / \mathrm{SiO}_{2}-\mathrm{P}$ into various solutions.

$\mathrm{RE}(\mathrm{III})$ ion $^{17)}$. The wash solution was supplied to discharge nonextracted elements from the column, and then $1 \mathrm{M} \mathrm{H}_{2} \mathrm{SO}_{4}$ solution was supplied to discharge the loaded Sc. In this experiment, excess volume of the wash solution and the eluent were supplied to properly discharge REs from the column.

Elution curves of all REs obtained by the column separation experiment are shown in Fig. 4. All REs except for Sc were not adsorbed by the column and were eluted with the feed solution, and this feature was exactly expected from the $K_{\mathrm{d}}$ profile. Small amount of Sc leaked from the column after the mobile phase was switched from the feed solution to the wash solution. Since the amount of HONTA must be enough for adsorption of all supplied Sc, some part of impregnated HONTA might not contribute to $\mathrm{Sc}$ extraction. Adsorption/elution performance often influenced by the amount of impregnated extractant ${ }^{20)}$, therefore composition of the adsorbent might be desirable to be improved for efficient use of the extractant.

Concentrated Sc solution was discharged from the column 
just after supplying the eluent, and Sc was not detected in the effluent after suppling $30 \mathrm{BV}$ of the eluent. If the product is set at initial few BV less than $25 \mathrm{BV}$ after switching to the eluent, the concentration of $\mathrm{Sc}$ in the product will be larger than the feed solution. This result implies that the extraction chromatography process can achieve separation and concentration of Sc simultaneously.

All supplied Sc was recovered in fractions of the wash solution and the eluent, and $97 \%$ was found in the first $30 \mathrm{BV}$ of the eluent. Amount of the leaked Sc in the wash solution might possible to be reduced by optimizing in composition of the adsorbent an in volume of the feed solution. If some fractions of the wash solution is added to the final product solution, recovery ratio of $\mathrm{Sc}$ increases while some other REs mixed with the Sc product. Figure 5 shows the purity of Sc plotted as function of the recovery ratio of Sc. About $97 \%$ of Sc was recovered with $99.9 \%$ purity, however the purity decreased to be about $98 \%$ if the recovery ratio is required to be more than $99.5 \%$. An appropriate specification of the Sc product solution has to be determined by requirements from its application.

Material balances of all REs are $100 \%$ and any elements did not remained inside the column after the operation, thus the column is possible to be used repeatedly. In order to evaluate application limit of the column precisely, not only repeated separation experiments but also investigation on durability of the HONTA $/ \mathrm{SiO}_{2}-\mathrm{P}$ adsorbent are required. As the adsorbent contacts with strong acids during the process, degradation of the adsorbent by decomposition and leaching of the HONTA extractant should be considered.

Table 1 Experimental condition of the column separation experiment.

\begin{tabular}{lll}
\hline & \multicolumn{2}{l}{$\begin{array}{l}\text { Flow velocity: } 1 \mathrm{~cm} / \mathrm{min} \\
\text { Temperature of the column: } 298 \mathrm{~K}\end{array}$} \\
\cline { 2 - 3 } & Solution & Amount \\
\hline \hline Conditioning & $4 \mathrm{M} \mathrm{HNO}_{3}$ & $5 \mathrm{BV}$ \\
\hline Feed & $\mathrm{RE}(\mathrm{III})$ in $4 \mathrm{M} \mathrm{HNO}_{3}$ & $25 \mathrm{BV}$ \\
\hline Wash solution & $4 \mathrm{M} \mathrm{HNO}_{3}$ & $10 \mathrm{BV}$ \\
\hline Eluent & $1 \mathrm{M} \mathrm{H}_{2} \mathrm{SO}_{4}$ & $50 \mathrm{BV}$ \\
\hline
\end{tabular}

\section{Summary}

New selective Sc recovery process flow using combination of the extraction chromatography technology and HONTA extractant was designed and performance of the process was experimentally demonstrated. Sc in nitric acid solution containing all rare earth elements was selectively adsorbed by HONTA impregnated adsorbent and successfully recovered into sulfuric acid solution. About $97 \%$ recovery ratio of Sc with $99.9 \%$ purity was achieved by a column separation test, and repeated use of the column must be possible. Further optimization in the adsorbent and in the flow-sheet are expected to improve the performance.

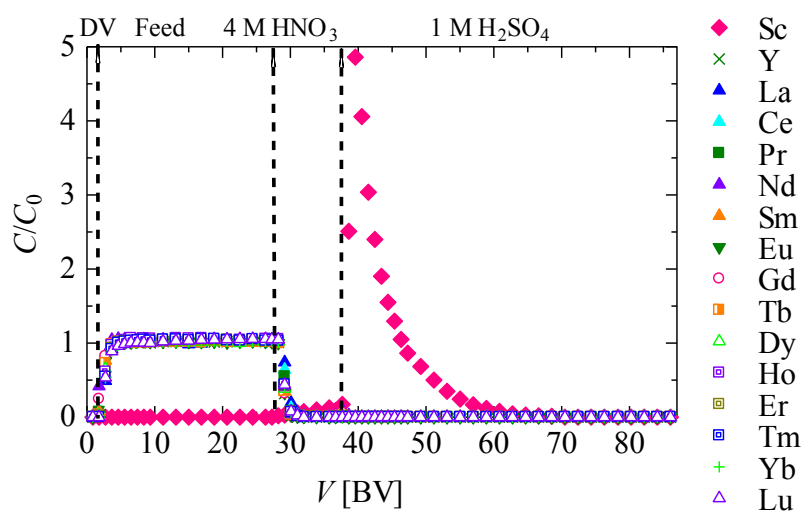

Fig. 4 Elution curves of REs obtained by the column separation experiment.

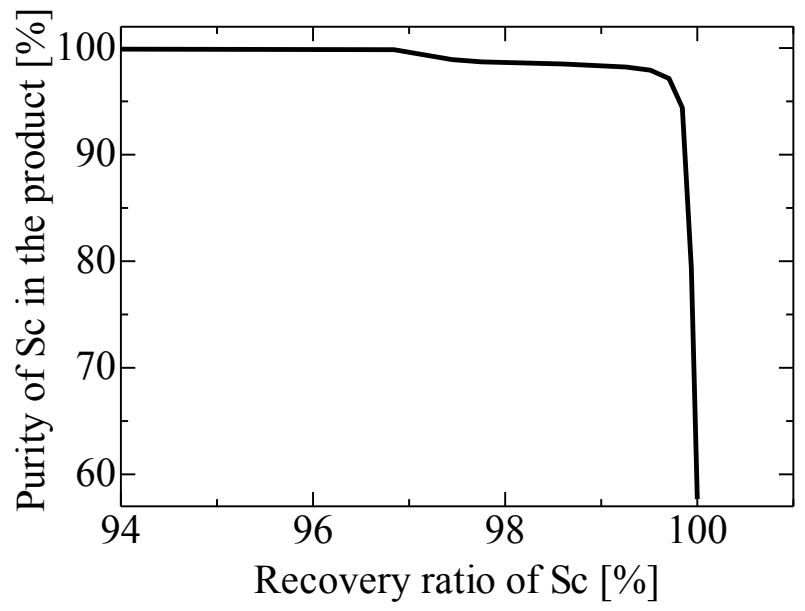

Fig. 5 Purity of Sc in the product plotted as function of recovery ratio.

\section{References}

1) M. Distaso and E. Quaranta, J. Catalysis, 253, 278 (2008).

2) C. Wang, G. Luo, M. Nishiura, G. Song, A. Tamamoto, Y. Luo and Z. Hou, Sci. Adv., 3, e1701011 (2017).

3) J. Röyset, Metall. Sci. Technol., 25, 2, 11 (2007).

4) R. Sawell and C. L. Jensen, Metall. Trans. A, 21, 421 (1990).

5) F. Rainer, W. H. Lowdermild, D. Milam, T. Tuttle Hart, T. L. Lichtenstein and C. K. Carniglia, Appl. Opt. 21, 3685 (1982).

6) B. Onghena, C. R. Borra, T. V. Ferven and K. Binnemans, Sep. Purif. Technol.,176, 208 (2017).

7) H. Nie, Y. Wang, Y. Wang, Z. Zhao, Y. Dong and X. Sun, Hydrometallurgy, 175, 117 (2018).

8) Y. Chen, H. Wang, Y. Pei and J. Wang, Sep. Purif. Technol.,178, 261 (2017). 
9) F. Basuki, N. Seko and M. Tamada, J. Ion Exchange, 21, 3, 127 (2010).

10) Y. Koma, Y. Sano, K. Nomura, S. Watanabe, T. Matsumura and Y. Morita, Proc. 11th IEMPT, San Francisco, USA, November 1-4, 2010, IV-4 (2010).

11) S. Watanabe, I. Goto, K. Nomura, Y. Sano and Y. Koma, Energy Procedia 7, 449 (2011).

12) S. Watanabe, K. Nomura, S. Kitawaki, A. Shibata, H. Kofuji, Y. Sano and M. Takeuchi, Procedia Chemistry, 21, 101 (2016).

13) S. Watanabe, I. Goto, Y. Sano and Y. Koma, J. Eng. Gas Turbines Power, 132, 102903 (2010).

14) S. Watanabe, Y. Sano, K. Nomura, Y. Koma and Y. Okamoto, EPJ Nuclear Sci. Technol. 1,9 (2015).

15) S. Watanabe, T. Senzaki, A. Shibata, K. Nomura, Y. Koma and Y. Nakajima, Proc. Global 2011, Chiba, Japan, December 11-16, 2011, 387433 (2011).

16) Y. Sasaki, Y. Tsubata, Y. Kitatsuji and Y. Morita, Chem. Lett., 42, 91 (2013).

17) Y. Wei, M. Kumagai and Y. Takashima, Nucl. Technol., 132, 413 (2000).

18) Y. Sano, S. Watanabe, H. Matsuura, K. Nagoshi and T. Arai, J. Nucl. Sci. Technol., 54, 10, 1058 (2017).

19) S.A. Cotton, Polyhedron 18, 1691 (1999).

20) S. Watanabe, T. Arai, T. Ogawa, M. Takizawa, K. Sano, K. Nomura and Y. Koma, Procedia Chemistry, 7, 411 (2012). 